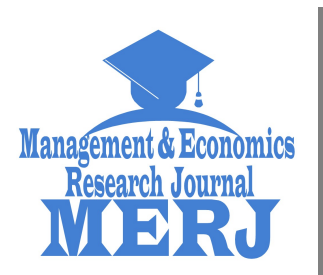

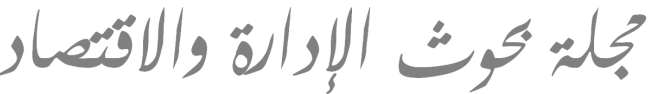

Management \& Economics Research Journal

Vol. 1 No. 2 (2019): June

المجلد 1 العدد 2 (2019): جوان

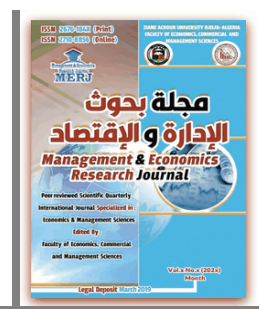

do https://doi.org/10.48100/merj.v1i2.30

Check for updates

\section{دور الصيانة في تخفيض التكاليف المتعلقة بتعطل الآلات دراسة حالة: مؤسسـة}

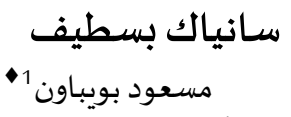

مسعود بويباون)

bouibam@yahoo.fr ماجستير، جامعة العربي بن مهيدي، أم البواقي (الجزائر)، الإيميل،

The Role of Maintenance in Reducing Costs related to Machine Failure: A Case Study of Saniak Company Setif

Messaoud Bouiban ${ }^{1}$

${ }^{1}$ Larbi Ben M'hidi University, Oum El Bouaghi (Algeria)

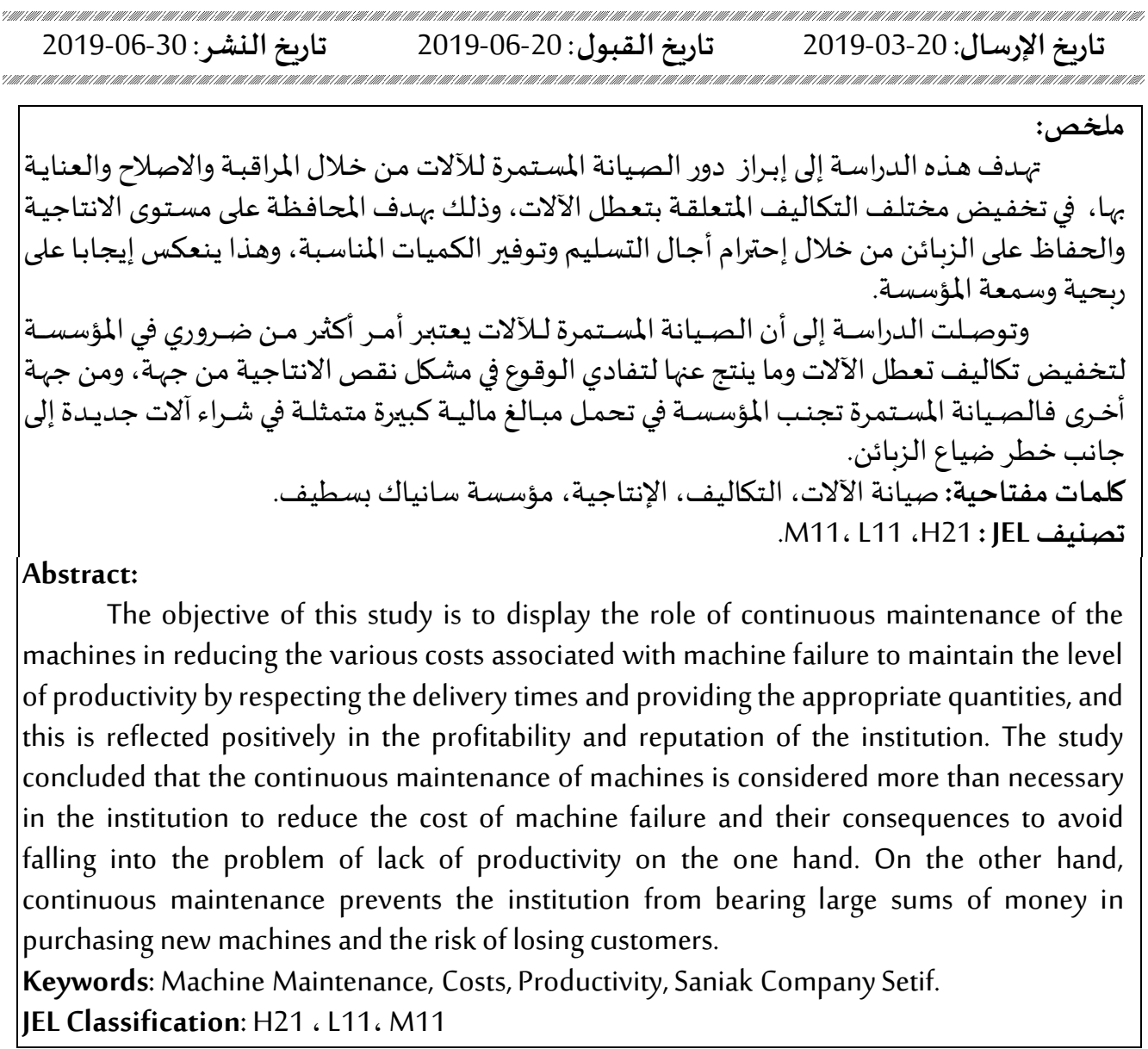

• المؤلف المراسل: مسعود بويباون، الإيميل: bouibam@yahoo.fr 


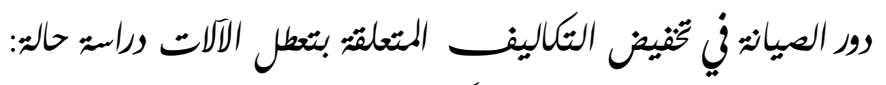

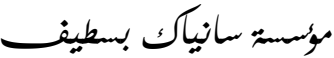

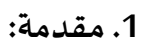

تشهد الكثير من المؤسسات الجزائرية إنخفاضا في مستوى الإنتاجية مقارنة مع الكمية المعيارية

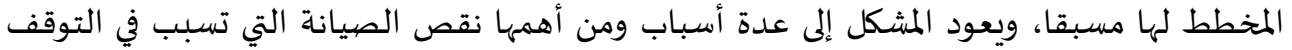

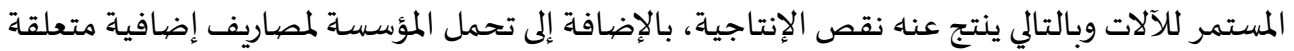

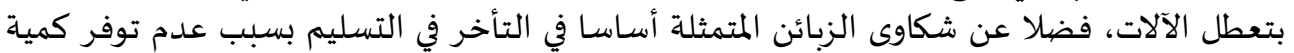
المنتجات المتفق عليها.

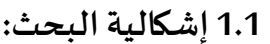

ومما سبق برزت معالم المشكلة التي تسعى هذه الدراسـة للإجابة عنها من خلال طرح التساؤل التالي:

ما هو دور صيانة الآلات في تخفيض التكاليف المتعلقة بتعطل الآلات؟

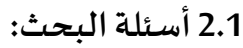

إنطلاقا من إشكالية البحث يمكن وضع الأسئلة التالية:

- هل تشكل صيانة الآلات تكلفة للمؤسسـة أم أنها تجنها الوقوع في تحمل تكاليف أكبر ؟.

- هل رفع مستوى الإنتاجية له علاقة بزيادة ربحية المؤسسة ؟. - ما هو واقع صيانة الآلات في المؤسسة محل الدراسة ؟.

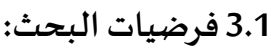

إن الإجابة عن هذا التسـاؤل يقودنا بالضيرورة لوضع الفرضيات التالية: - تعتبر صيانة الآلات من الاجراءات المهمة لتجنب توقف الآلات عن الانتاج.

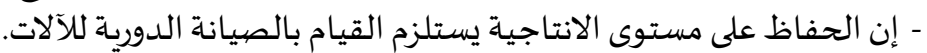

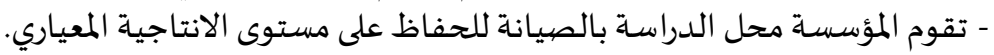

4.1 أهمية البحث:

يكمن أهمية البحث في ما يلي:

- إبراز دور صيانة الآلات بمدى مستوى الإي: الاهتمام الذي تقوم به المؤسسـة في هذا المجال.

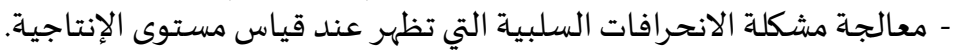

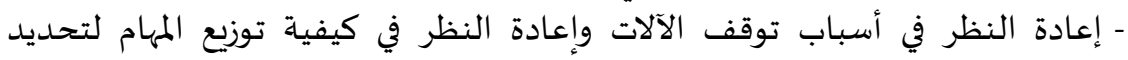

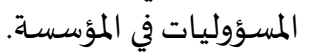

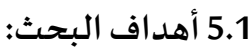

يهدف البحث إلى ما يلي: ئح:

- التحسيس بمدى دور صيانة الآلات في تخفيض التكاليف المتعلقة بتعبط الآلآلات لرفع

مستوى الإنتاجية.

- يسعى البحث في إيجاد علاقة زيادة الإنتاجية بزيادة الإنيادة ربحية المؤسسـة.

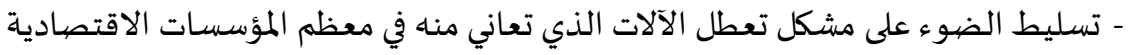

$$
\text { الجزائرية. }
$$

6.1 منهجية البحث:

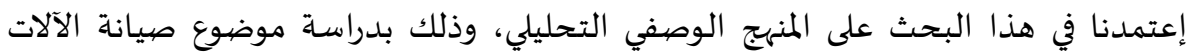

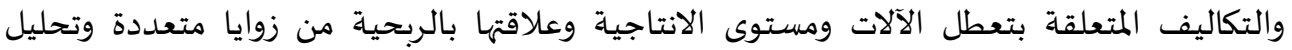

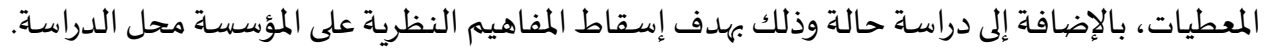


المجلل: 01 - العد: 02 (جوان 2019 (2019)

يعتبر تعطل الآلات من بين المشاكل الحديثة التي تواجه المؤسسة بسبب إستخدامها لألاتلات ذات

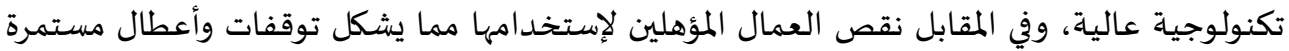

تعتبر وظيفة الصيانة ضرورية في المؤسسة وتنقسم الصيانة إلى نوعين هما: (ايمن، 2019،

صفحة 02)

- الصيانة الوقائية: وتعرف على أنها مجموعة من الأنشطة والإجراءات التي تتخذها إدارة الصيانة،

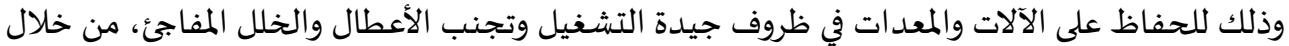

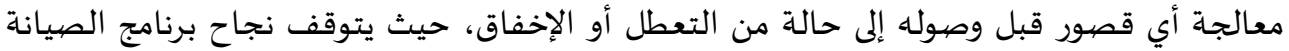

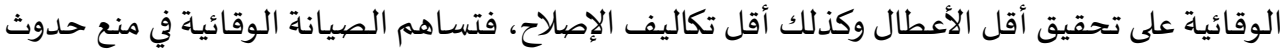

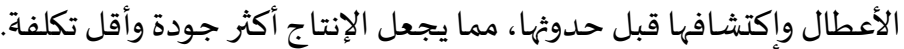

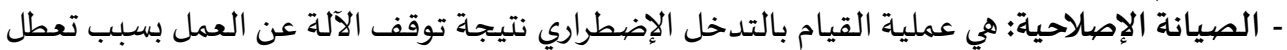

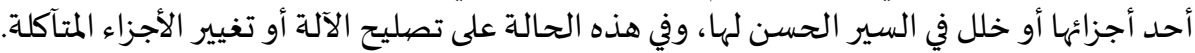

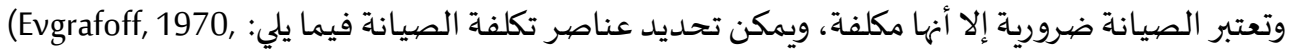

ل أعباء تسيير المصلحة المختصية بالصيانة في المؤسسة.

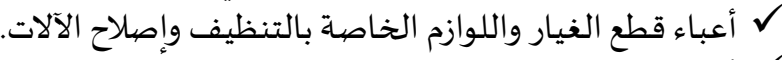

$$
\checkmark
$$

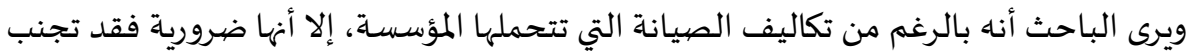

المؤسسة الوقوع في تكاليف أكثر.

2.2 تعريف تعطل الآلات:

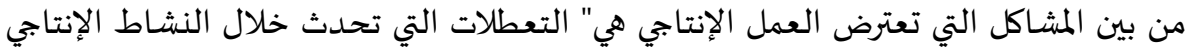

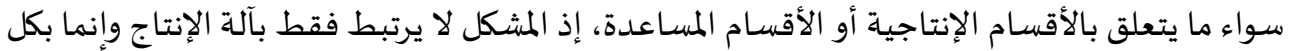

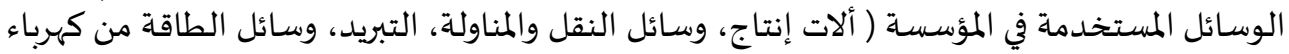

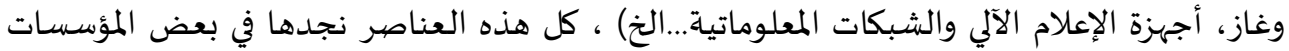

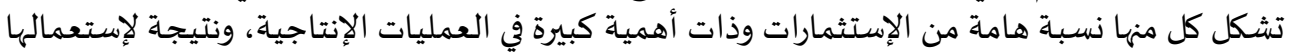

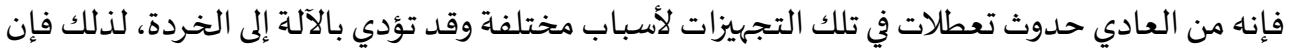

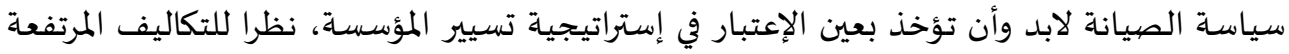

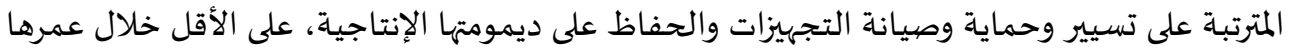

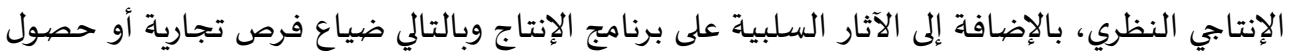

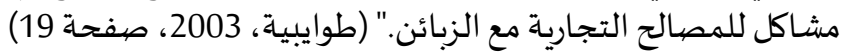

3.2 دور الصيانة في تخفيض التكاليف المتعلقة بتعطل الآلات:

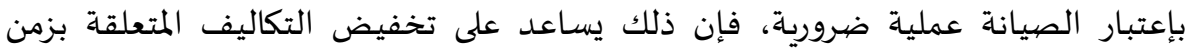

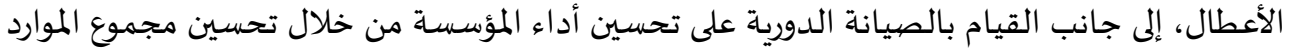

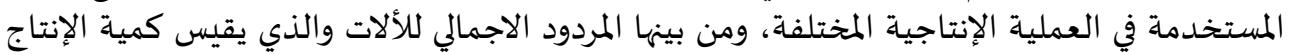

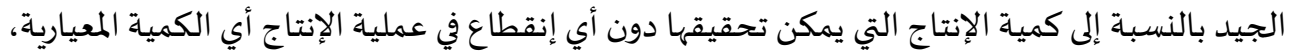

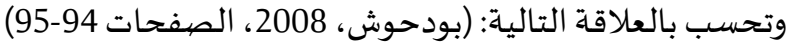




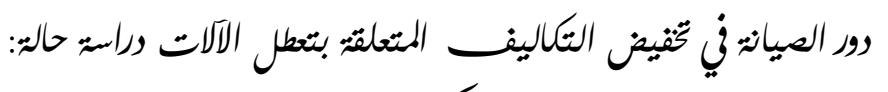

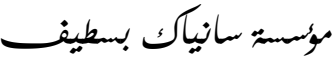

$P-D E F$

N

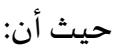

: معدل المردود الإجمالي للآلات. TRG

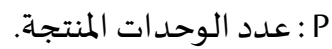

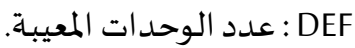

N عدد الوحدات المعيارية الممكن إنتاجها.

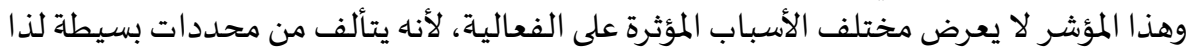

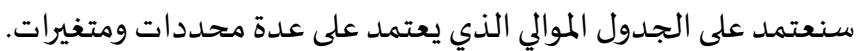

جدول رقم (01): يبين نموذج حساب زمن التشغيل الفعال للألة

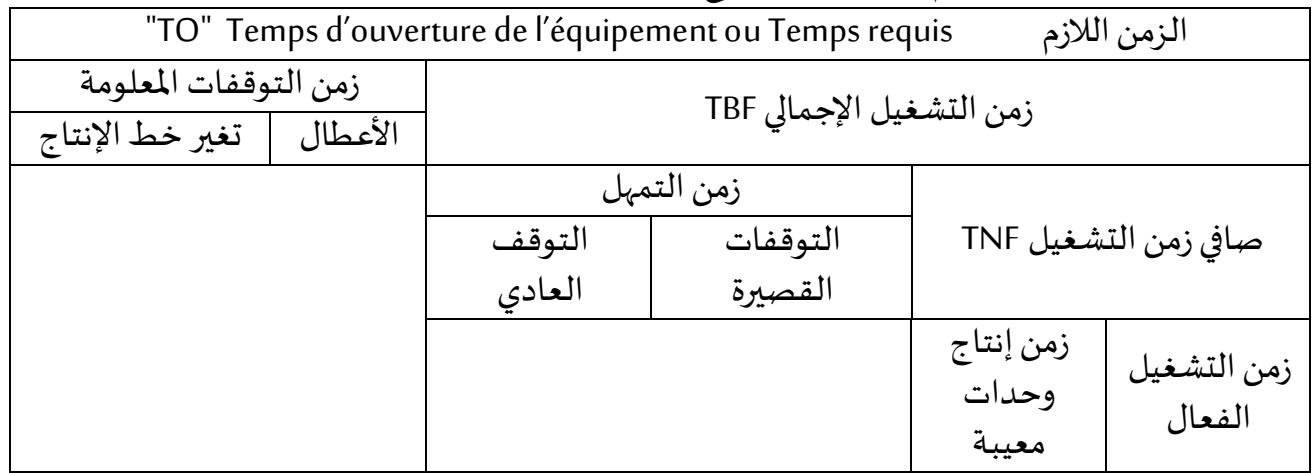

المصبدر: (بودحوش، 2008، صفحة 95)

من خلال الجدول أعلاه نلاحظ مختلف الأوقات المتعلقة بإنتاج منتوج معين، فكلما زادت دقة

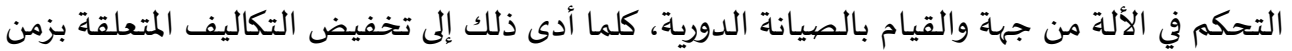

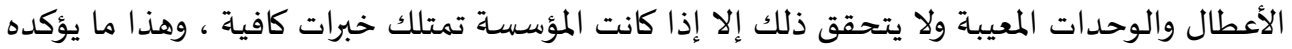

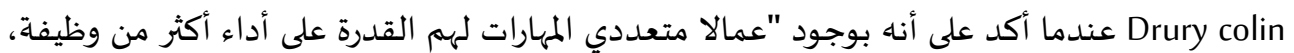

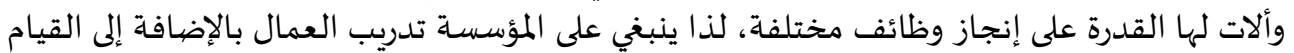

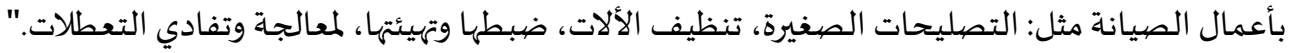

(colin, 2001, pp. 471-472)

3. 1 الانتاجية:

تعتبر الإنتاجية في المؤسسة بمثابة "معيارا لقياس مدى كفاءة المؤسسة في إستخدام مواردها

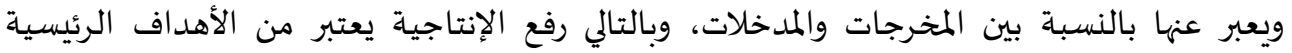

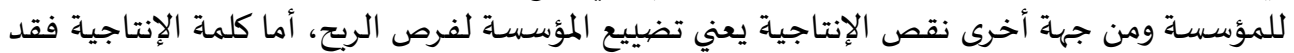

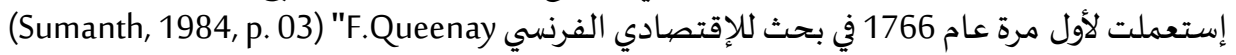




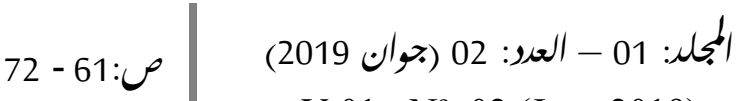

$$
\begin{aligned}
& \text { V:01 - No :02 (June 2019) }
\end{aligned}
$$

وتعرف الإنتاجية على أنها " مقياس للتشغيل الإقتصادي للطاقات المتاحة." (عبدالحكيم، 1990،

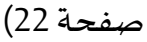

وتعرف الإنتاجية أيضا على أنها " العلاقة بين الموارد "inputs" المستخدمة في العملية الإنتاجية

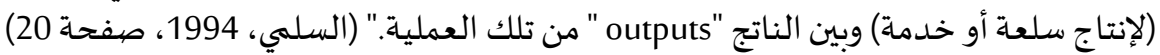
2.3 حساب مؤشر الانتاجية:

ويحسب مؤشر الانتاجية كما يلي: (عليان، 1998، صفحة المجاتية 558)

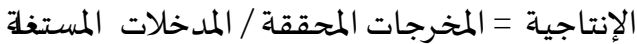

ولمؤشر الإنتاجية قياسات عديدة في المؤسسة كمات يوضحة /لمدخه الجدات المستفالئ التالي:

جدول رقم (02): يبين مؤشرات قياس الإنتاجية

\begin{tabular}{|c|c|}
\hline معادلات الإنتاجية & طرق حسـاب الإنتاجية \\
\hline متوسط إنتاجية العمل = كمية الإنتاج / متوسط عدد العمال & طريقة كمية الإنتاج \\
\hline متوسط إنتاجية العمل = إجمالي قيمة الإنتاج / متوسط عدد العمال & طريقة قيمة الإنتاج \\
\hline متوسط إنتاجية العمل = القيمة المضافة / متوسط عدد العمال & طريقة القيمة المضافة \\
\hline إنتاجية الدينار من الأجور= قيمة إنتاج الفترة / قيمة الأجور لنفس الفترة & طريقة الأجور \\
\hline
\end{tabular}

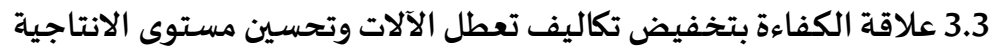

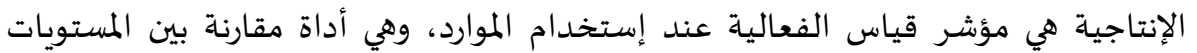

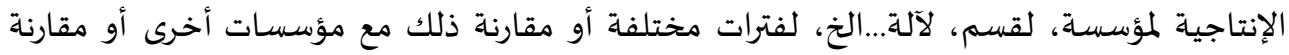

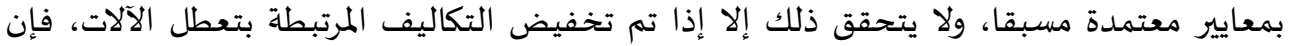

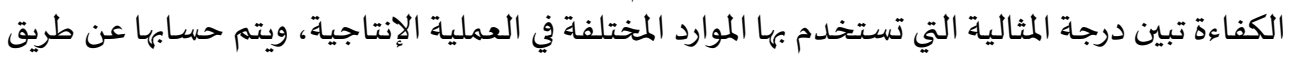

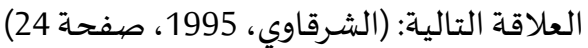

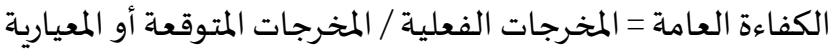

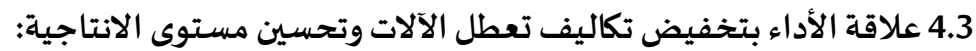

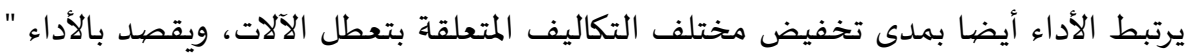

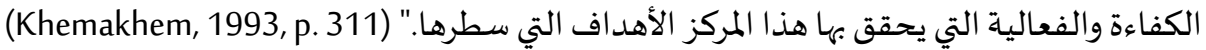

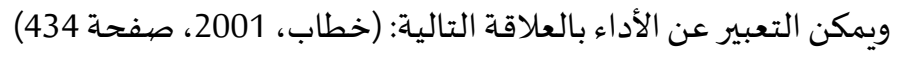
الأداء = الفعالية × الكفاءة المباء

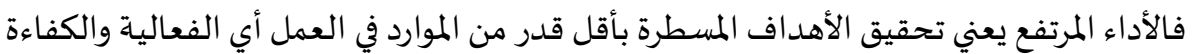

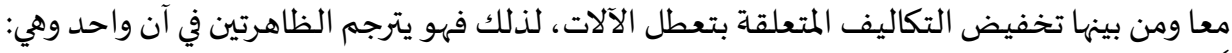
درجة بلوغ الهدف أو الأهداف المحددة المنعة ( الفعالية). الطريقة التي تم بها تحقيق النتائج (الكفاءة الماءة).

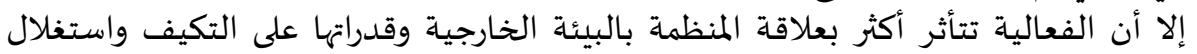

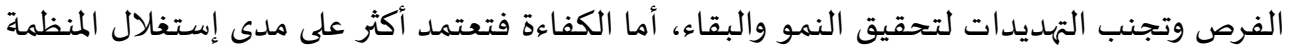




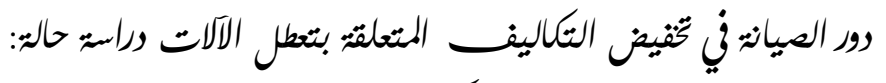

للموارد برشادة، وبما أن الأداء يشمل البعدين (الكفاءة والفعالية) فإنه يجب على المنظمة التحيين المستمر لوضع إستراتجيات مدروسة لتحسين الاداء بثلمل البعدين الانتاجية.

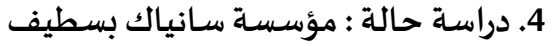

(1.4 تعريف مختصر بالمؤسسة:

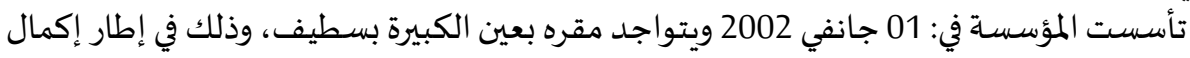

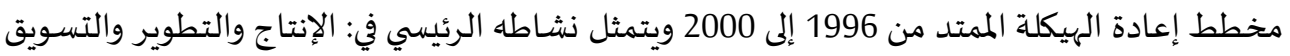

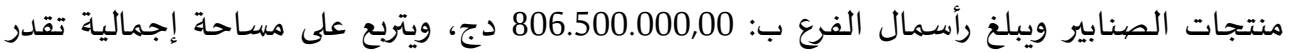

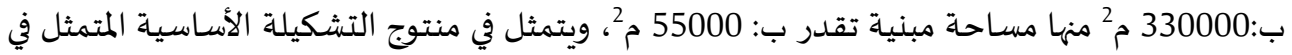

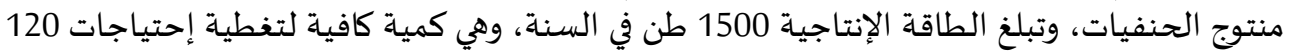
ألف مسكن في السنة.

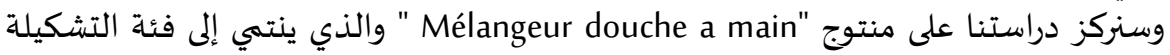

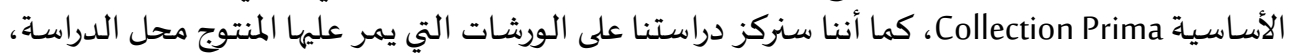
وكان سبب إختيارنا لهذا المنتوج دون غيره للأسباب التالية:

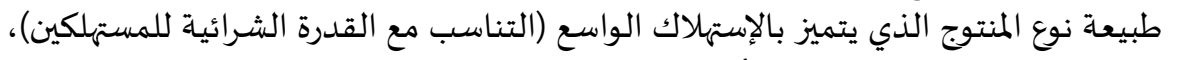

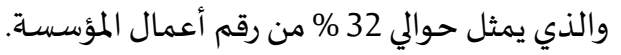

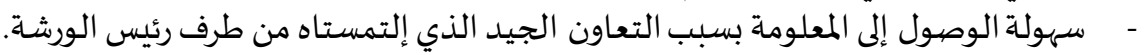

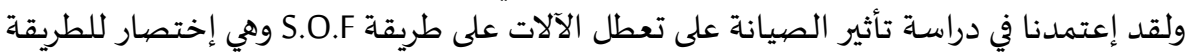

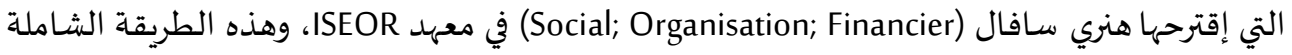
ترتكز على ثلاثة مقاييس وهي: المقياس الاجتماعي: ويكون مصدي: الإندا الحصول عليها عند دراسة حالة عن طريق الملاحظة وأسلوب

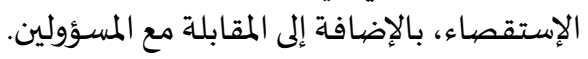

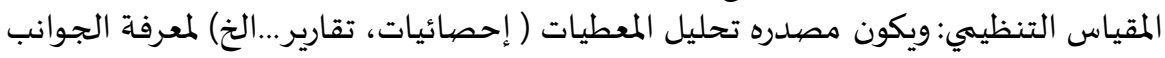

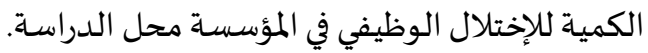

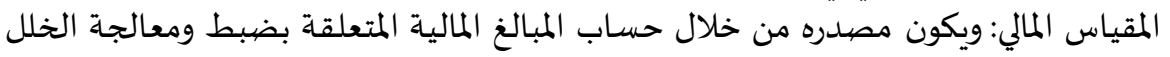

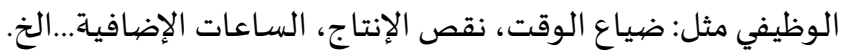

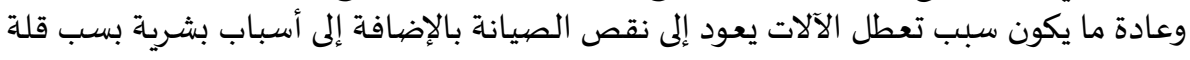

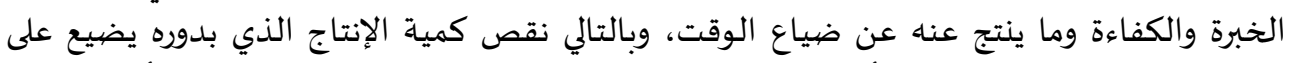

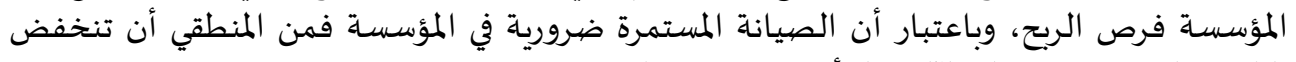

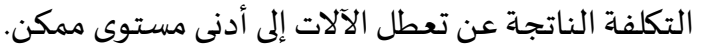

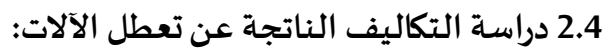

وسنعتمد على المعايير الثلاثة المشار الهيا سابقا وهي المقياس الاجتماعي والمقياس التنظيمي والمقياس المالي. أولا: المقياس الإجتماعي من خلال دراستنا التطبيقية وبالاعتماد على أسلوب الملاحظة لاحظنا عدد كبير من الآلات

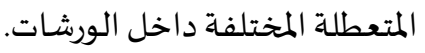

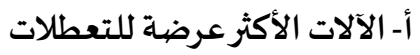

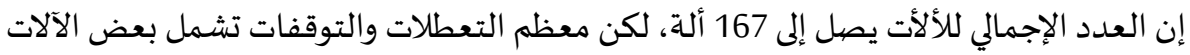
بصورة كبيرة حسب ما يوضحده الجدول الموالي لالي. 
المجلل: 01 - العر: 02 (جوان 2019 ( 2019)

جدول رقم (03): يبن أهم الآلات الأكثر تعطلا في المؤسسة

\begin{tabular}{|c|c|c|}
\hline التعيين & 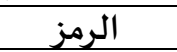 & الورشـة \\
\hline Perceuse ALMO & D6234011 & $350 \mathrm{~A}$ \\
\hline Presse à découper type:GHP40,400KN & D6415031 & $380 \mathrm{E}$ \\
\hline Machine à fabriquer les noyaux ( $\mathrm{H} 2,5 \mathrm{Co})$ & D6592014 & \multirow{3}{*}{$450 \mathrm{~A}$} \\
\hline Machine à couler en coquilles & D6585015 & \\
\hline MACHINE HYDROLIQUE DE COULEE IMR C55H & D6585033 & \\
\hline Grenailleuse TAMBRO 1000 EXK & D6533011 & \multirow{5}{*}{$450 \mathrm{C}$} \\
\hline Tronçonneuse manuelle DIAM 400 & D6283031 & \\
\hline Meuleuse à bande SMF 350 & D6331029 & \\
\hline GRENALLEUSE TURBOTECNICA & D6533065 & \\
\hline Emerisseuse à bande SM-F 350 & D6331051 & \\
\hline Tour revolver HERBERT & D6210021 & \multirow{3}{*}{$451 \mathrm{~A}$} \\
\hline Machine à briqueter $300 \mathrm{KN} 1000 \mathrm{KGS} / \mathrm{H}$ & D6423011 & \\
\hline Scie circulaire automatique RYKART (RKA 53S-600) & D6282015 & \\
\hline Machine spéc. à 4 broches 4 A-11.2 + (rénovation) & D6373015 & \multirow{5}{*}{ 470B } \\
\hline Perceuse verticale VARIA 23 & D6234022 & \\
\hline Fraiseuse universelle F 7-PNL-Z SV & D6264045 & \\
\hline Tour revolver 149x152 FLASHCAP 2 & D6216012 & \\
\hline Tour automatique $102 \mathrm{HP}$ & D6229011 & \\
\hline DAF 6.4 & D6373030 & \multirow{6}{*}{$470 \mathrm{~F}$} \\
\hline ZAF 6.4 & D6373031 & \\
\hline Tour automatique multi-broches KS 25 + Rénovation & D6224035 & \\
\hline Emerisseuse à bande SM-F 350 & D6331025 & \\
\hline Meuleuse à bande L 60 & D6331027 & \\
\hline Polisseuse à bande stahl SMF 350 & D6331071 & \\
\hline APPAREIL DE MARQUAGE:TD412-12W YAG & D6146055 & \multirow{3}{*}{$490 \mathrm{~A}$} \\
\hline Perceuse $6 \mathrm{~K}$ & D6232013 & \\
\hline BANC DE MONTAGE FAB 6-4 BAV FE & D7101010 & \\
\hline
\end{tabular}

المصبدر: من إعداد الباحث بالاعتماد على وثائق المؤسسة

نلاحظ من خلال الجدول العدد الهائل من الآلات المعطلة التي تكبد المؤسسة خسائر كبيرة تتمثل

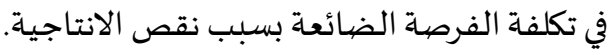
ب- إحصائيات تعطل الآلات في المؤسسة

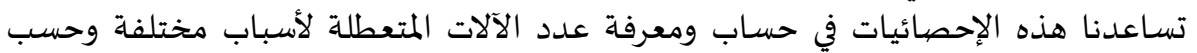
السنوات، ويمكن تلخيصها في الجدول الموالي. 


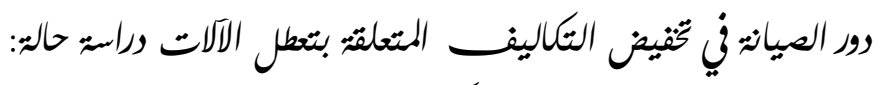
مؤسسية سانيكت بسطينس

جدول رقم (04): يبين السـاعات الضائعة بسبب تعطل الآلات.

\begin{tabular}{|c|c|c|c|c|c|c|}
\hline التغير\%\% & 2017 & التغير\% & 2016 & 2015 & 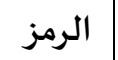 & السبب \\
\hline$-25,66$ & 4107,78 & $-22,69$ & 4271,64 & 5525,65 & IMP 05 & نقص قطع الغيار \\
\hline 17,46 & 20206,27 & $-24,30$ & 13022,70 & 17202,47 & IMP 10 & عدم مطابقة قطع الغيار \\
\hline$-13,34$ & 7653,20 & 26,14 & 11140,06 & 8831,55 & IMP 11 & أسباب ميكانيكية \\
\hline 16,93 & 45388,34 & 7,31 & 41652,36 & 38815,29 & & المجموع \\
\hline
\end{tabular}

المصبدر: من إعداد الباحث بالاعتماد على وثائق المؤسسة

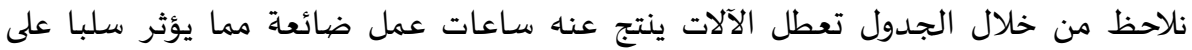

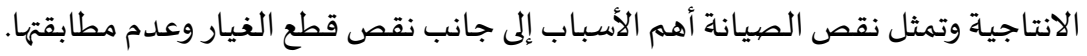

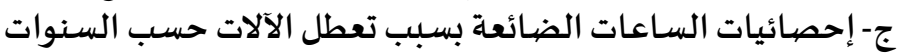

من خلال الجدول وللتوضيح أكثر يمكننا تمثيل الإحصيائيات على شكل الماتل أعمدة بيانية من خلال

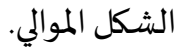

شكل رقم (01): يوضيح الساعات الضيائعة بسبب تعطل الآلات حسب السنوات

اتعة بسبب التعطلات

50000,00

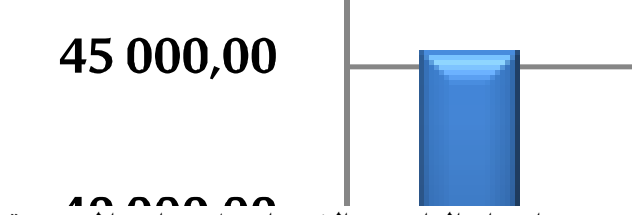

المصيدر: من إعداد الباحث بالاعتماد على وثثائقَ المؤَسسة

من خلال الشكل البياني نلاحظ أن الساعات الضائعة بسبب تعطل الآلات في زيادة مستمرة

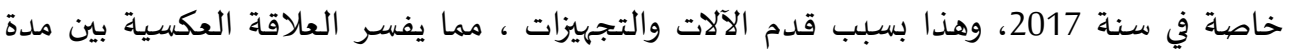

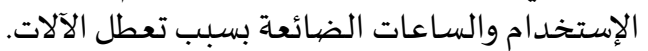

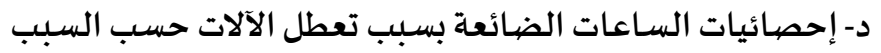
ومن خلال الجدول السابق، يمكننا تمثيل الإحصائيات على شكل أعمدة بيات بيانية من خلال الشكل 


$$
\begin{aligned}
& \text { المجلل: } 01 \text { - العرد: } 02 \text { (جوان } 2019 \text { ( 2019) } \\
& \mathrm{V}: 01 \text { - No :02 (June 2019) }
\end{aligned}
$$

جلن: تحوث الإراة والاقتصار

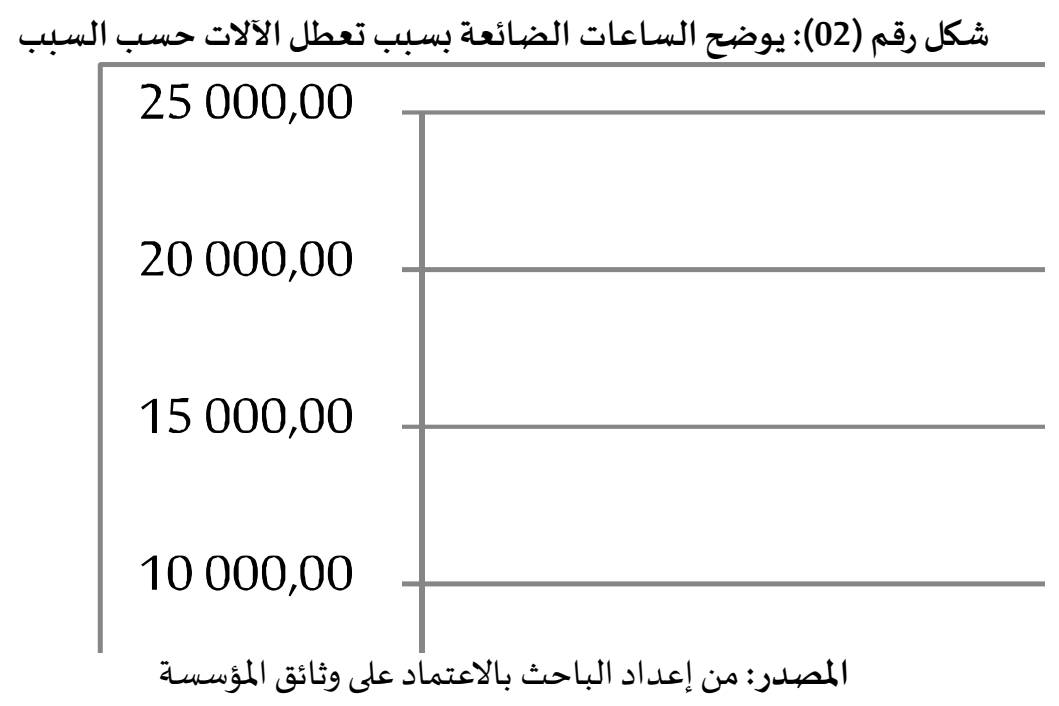

من خلال الشكل البياني نلاحظ أن سبب الساعات الضائعة بسبب تعطل الآلات يعود بالدرجة

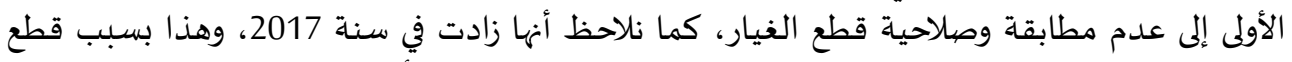

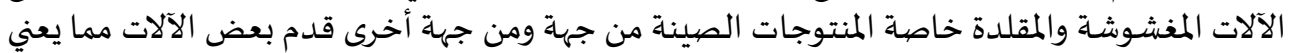

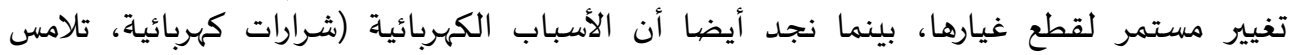

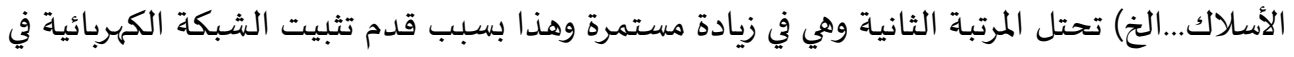
ويتمثل في مختلف الإجراءات المتخذة والتي من شأنها معالجـة الخلل للحفاظ المقاء على الإنتاج العادي

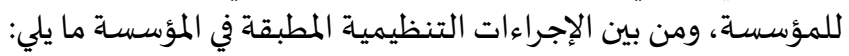

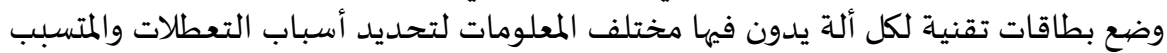

الصيانة الدورية للآلات خاصية القديمة منها.

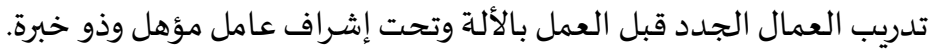
النظر في إعادة وضيع وترتيب الآلات. توفير المناخ الفزيائي المناسب.

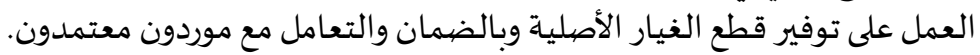

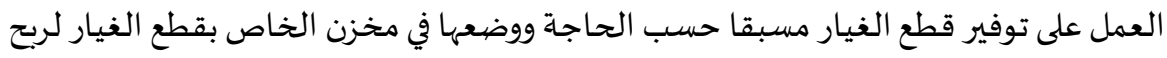

$$
\text { الوقت. }
$$

ثالثا: المقياس المالي ويمثل الترجمة النقدية لإجراءات معالجة الخلل الوظيفي المتعلق بمختلف التعطلات وحسب المبات

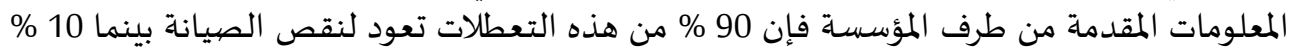
تعود لأسباب قاهرة وخارج السيطرة، وبالتالي يمكن حساب السماعات المات الضيائعة بسبب تعطل الآلات لسنة 


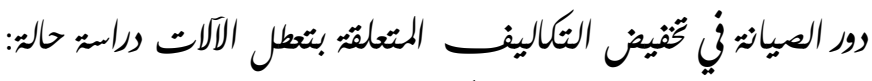

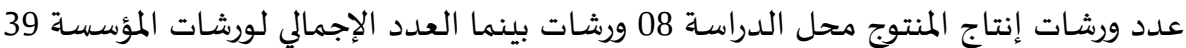

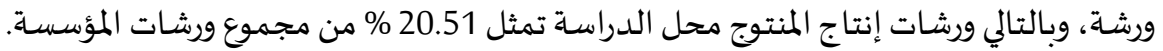

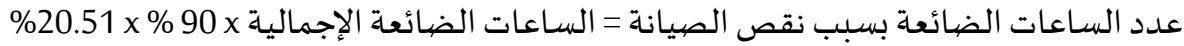
عدد الساعات الضائعة بسبب نقص الصيانة = 388,34 8378.23 = 20.51 x \% 90 x 45 ×

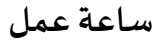

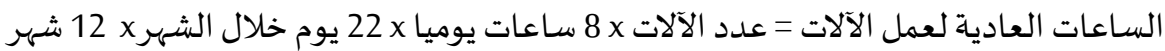

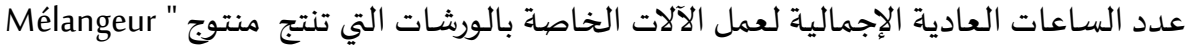
" douche a main

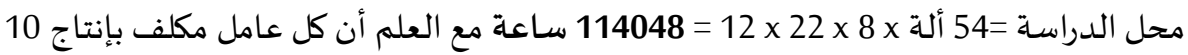

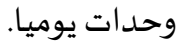

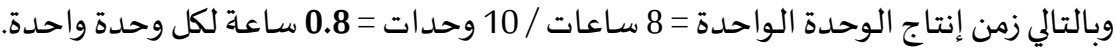

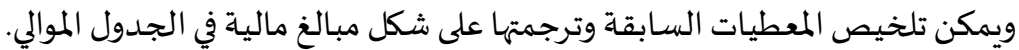

\section{جدول رقم (05): يبين التكاليف الناتجة تعطل الآلات}

\begin{tabular}{|c|c|}
\hline المبلغ دج & عناصر التكلفة \\
\hline 1722840.00 & 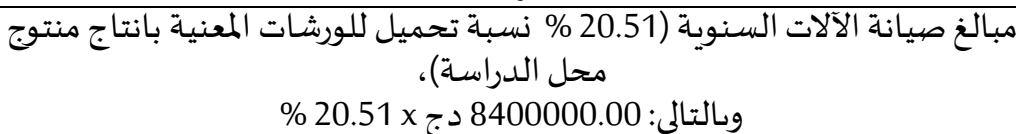 \\
\hline 1495614.59 دج & 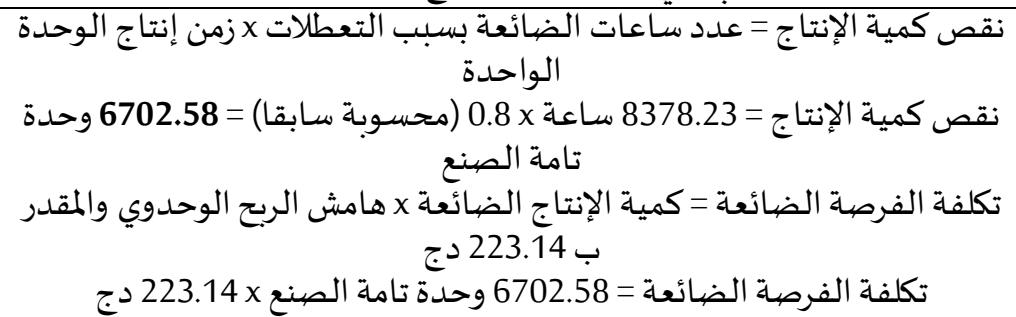 \\
\hline $3.218 .454,59$ & مجموع التكاليف l الناتجة عن تعطل الآلات \\
\hline
\end{tabular}

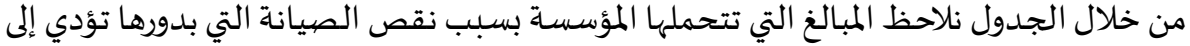

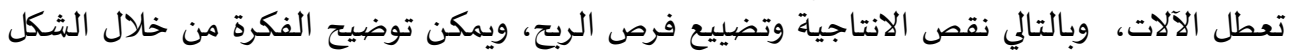
الموالي. 


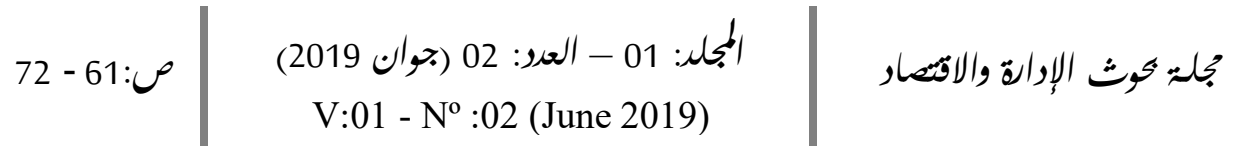

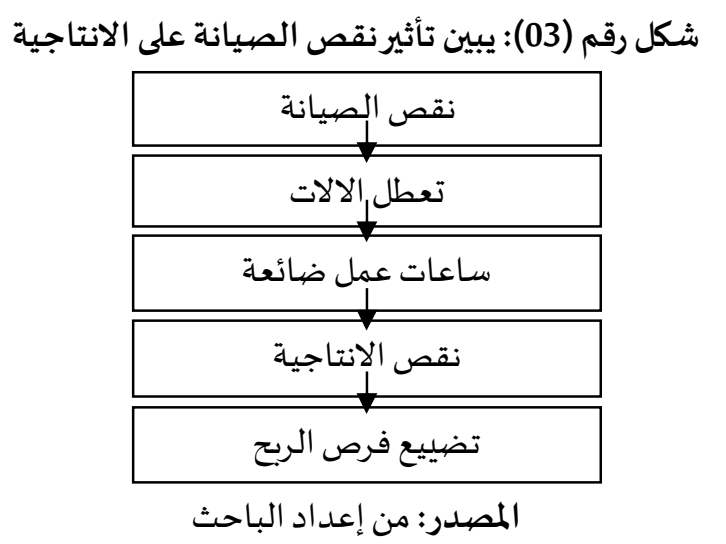

5. - 20 - تحليل النتائج:

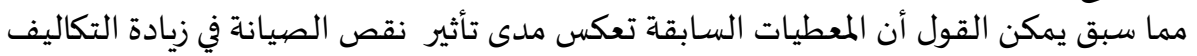

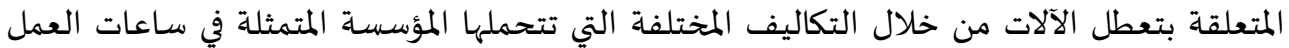

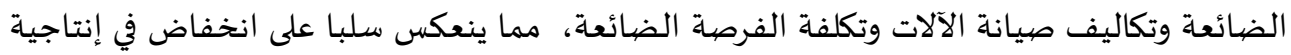

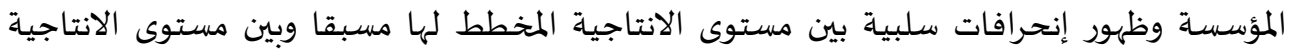

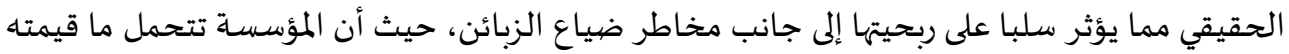

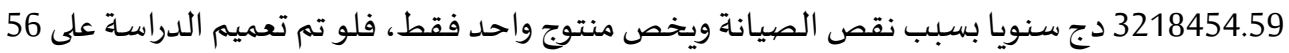
منتوج تنتجه المؤسسة مع افتراض تجانس المعطيات لتحصلنا على: 3.218.454,59 دج

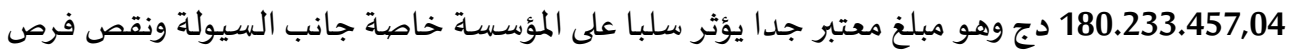

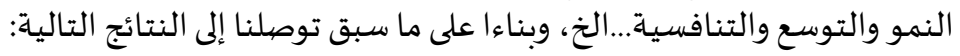

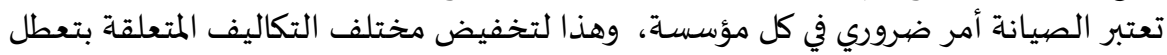

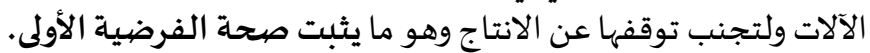

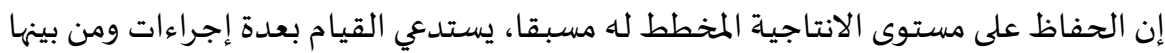

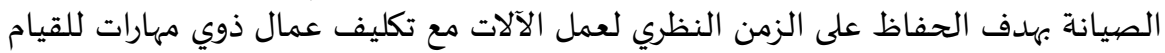

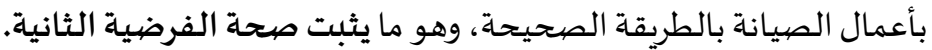

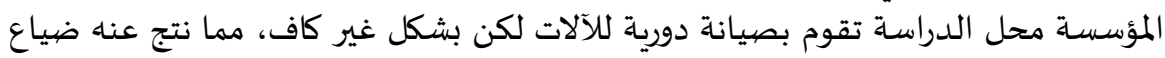

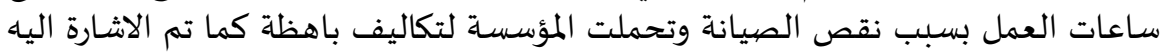
سابقا، مما نتج عنه إنحراف سلبي في مقدار الانتاجية، وهو ما ينفي صححة الفرضيت لموضية الثالثة.

6. الخلاصية:

لقد أوضحنا مفهوم الإنتاجية وأهمية تحسينها باعتبارها هدفا من بين الأهداف التي تسعى الته

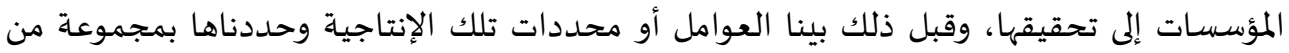

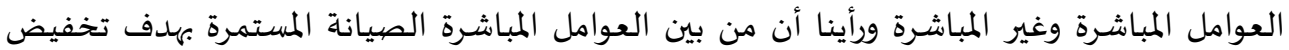

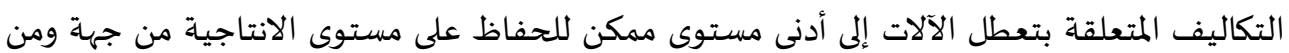

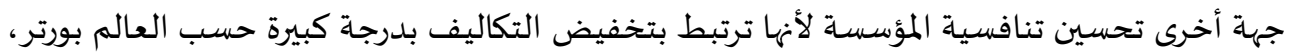

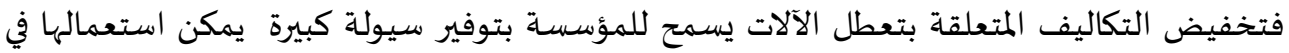

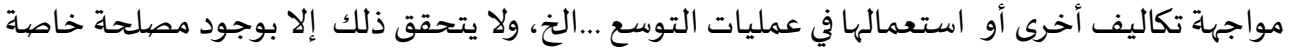

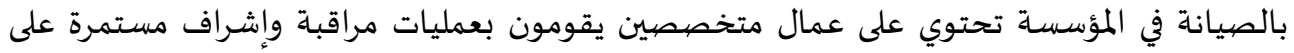




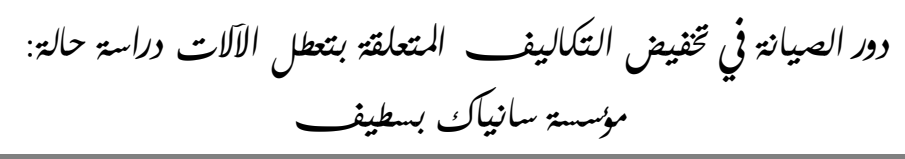

مختلف البالات مع إعداد بطاقات خاصة بالصيانة والمتابعة وحالة كل ألة على حدى لتسهيل عملية

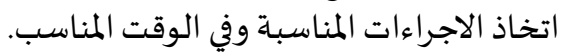

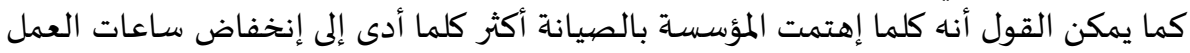

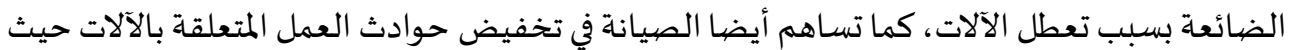

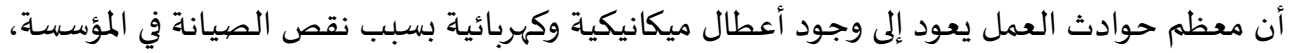

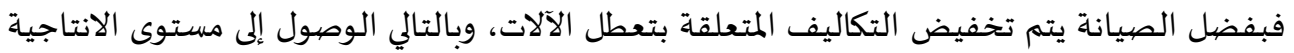

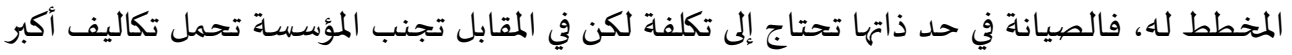

Colin, D. (2001). Management accounting for business decisions. London: Thomson Learning.

Evgrafoff, B. (1970). Systèmes de gestion de la production. France: édition Paris Sirey.

Khemakhem, A. (1993). La dynamique de contrôle de gestion. Paris: édition Dunod.

Sumanth, D. J. (1984). Productivity engineering and management. New York: McGraw mill book company.

إبر اهيم عبدالحكيم. (2003). (1990). الكفاية الإنتاجية في الصناعة العربية. مجلة التنمية الصناعية العربية، 9.

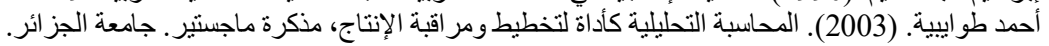

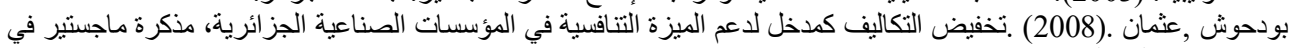
علوم التسيير .جامعة سكيكدة. خطاب عايدة .(2001) .,العولمة وإدارة الموارد البكة البشرية .القاهرة : دار الفكر العربي.

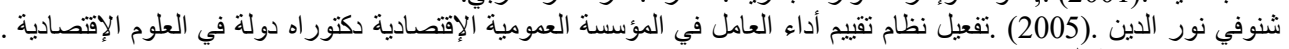
جامعة الجز ائر 3. علي السلمي. (1994). الإدارة الإتتاجية. القاهرة: مكتبة الإدارة الجديدة.

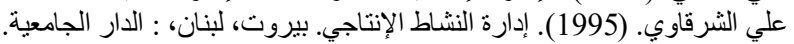

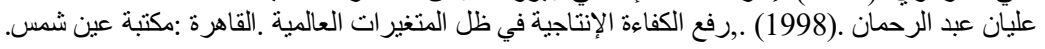

\section{References:}

Colin, D. (2001). Management accounting for business decisions. London: Thomson Learning.

Evgrafoff, B. (1970). Systèmes de gestion de la production. France: édition Paris Sirey.

Khemakhem, A. (1993). La dynamique de contrôle de gestion. Paris: édition Dunod.

Sumanth, D. J. (1984). Productivity engineering and management. New York: McGraw mill book company.

Abdelhakim, I. (1990). Productive efficiency in the Arab industry. Arab Industrial Development Journal, 9. [In Arabic]

Tawabeya, A. (2003). Analytical Accounting as a Tool for Production Planning and Control, (Master Thesis. Algiers University). [In Arabic]

Bodhouch, O. (2008). Cost reduction as an input to support the competitive advantage in Algerian industrial enterprises, (Master Thesis. Skikda University). [In Arabic]

Aida speech. (2001). Globalization and human resource management. Cairo: Dar Al-Fikr Alarabi. [In Arabic]

Shenoufi, N. (2005). Activating the employee performance evaluation system in the Economic Public Corporation. (PhD in Economic Sciences. Algiers3 University). [In Arabic]

Al-Soullami, A. (1994). Productive management. Cairo: Library of the New Administration. [In Arabic]

Al-Sharqawi, A. (1995). Managing productive activity. Beirut, Lebanon: University House. [In Arabic]

Alyan, A. (1998). Raise production efficiency in light of global changes. Cairo: Ain Shams Library. [In Arabic] 